\section{Calcificaciones pulmonares metastásicas en un trasplantado renal con un injerto con función renal adecuada. Caso clínico}

\author{
JORGE VEGA ${ }^{1,2,3}$, HELMUTH GOECKE ${ }^{1,2}$, \\ HUGO VALENZUELA ${ }^{4}$, MARIO SANTAMARINA ${ }^{5}$
}

\section{Metastatic pulmonary calcification in a patient with a functioning kidney allograft. Report of one case}

In renal transplants patients, metastatic pulmonary calcifications have been reported occasionally when the grafts are dysfunctional and rarely when they are functioning normally. We report a male who received a renal allograft in 1994 at the age of 61 years. Nineteen years later a routine chest $X$ ray showed diffuse infiltrates and a CT scan showed diffuse calcifications in both lungs. These were interpreted as metastatic pulmonary calcifications. The last available laboratory determinations were a serum creatinine of $1.4 \mathrm{mg} / \mathrm{dl}$ and urinary protein excretion of $255 \mathrm{mg} / 24$ hours. No further studies were done since the patient experienced a sudden death due to an acute myocardial infarction.

(Rev Med Chile 2013; 141: 1589-1593)

Key words: Kidney transplantation; Pulmonary calcification; Renal insufficiency.
${ }^{1}$ Escuela de Medicina, Universidad de Valparaíso, Valparaíso, Chile. ${ }^{2}$ Servicio de Medicina, Sección Nefrología, Hospital Naval A. Nef, Viña del Mar, Chile.

${ }^{3}$ Servicio de Medicina, Sección Nefrología, Hospital Dr. Gustavo Fricke, Viña del Mar, Chile. ${ }^{4}$ Servicio de Medicina, Hospital E. Pereira, Valparaíso, Chile. ${ }^{5}$ Servicio de Radiología, Hospital Naval A. Nef, Viña del Mar, Chile.

Recibido el 27 de junio de 2013, aceptado el 28 de octubre de 2013.

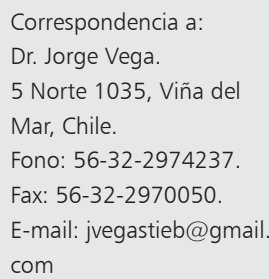

L a calcificación de tejidos blandos es frecuente en pacientes portadores de insuficiencia renal crónica (IRC) y terapia con hemodiálisis (HD), particularmente en los vasos sanguíneos. Estas calcificaciones pueden ocurrir en cualquier tejido, más frecuentemente en arterias coronarias, aorta, riñón, estómago y pulmón ${ }^{1-3}$. El depósito tisular de calcio predominantemente ocurre en los lugares de excreción ácida como estómago (ácido clorhídrico), riñón (ácido úrico), pulmón (ácido carbónico $)^{4}$.

Las calcificaciones pulmonares pueden ocurrir en un tejido previamente dañado (distróficas) o en tejido sano (metastásicas) ${ }^{5}$. Estas últimas son frecuentes en pacientes con IRC en HD, aunque muy rara vez se diagnostican en vida ${ }^{6-12}$. Las radiografías de tórax tienen una escasa sensibilidad para detectarlas ${ }^{10,12,17}$. En autopsias de pacientes con IRC en HD se encuentran calcificaciones pulmonares metastásicas (CPM) en $60-75 \%$ de ellos $^{13}$. La evolución clínica de las CPM habitualmente es benigna y a menudo asintomática. Otras veces existe tos no productiva y disnea de esfuerzo, ocasionalmente puede seguir un curso fulminante y fatal ${ }^{9,14,17} \mathrm{o}$ asociarse a calcifilaxis $\mathrm{y}$ necrosis pulmonar ${ }^{18}$.

Las CPM se han observado también en pacientes con hiperparatiroidismo primario $y$ secundario, hipercalcemias de otras causas, terapia endovenosa con calcio, mieloma múltiple, leucemias, sarcoidosis, intoxicación por vitamina $\mathrm{D}$, osteolisis masiva por metástasis, síndrome de leche-alcalinos, enfermedad de Paget, neoplasia endocrina múltiple ${ }^{9,19-21}$ y en trasplantados renales (TxR) con injertos disfuncionantes ${ }^{4,7,14,16,17,22,23}$. Rara vez se ha observado CPM en pacientes con trasplantes exitosos ${ }^{8,24,25}$.

Comunicamos un caso de CPM diagnosticada en un paciente trasplantado renal 19 años atrás con una buena función del injerto. 


\section{Caso clínico}

Paciente portador de IRC de etiología desconocida que estuvo en HD durante 2 años, sin complicaciones, salvo una polineuropatía periférica. Le fue trasplantado un riñón de su esposa a los 61 años de edad en octubre de 1994. La terapia inmunosupresora fue ciclosporina, azatioprina y prednisona. Se utilizó diltiazem y ketoconazol conjuntamente para reducir la dosis diaria de ciclosporina, por motivos económicos.

$\mathrm{Al}$ año del TxR la creatinina era $1,22 \mathrm{mg} / \mathrm{dl}$ y el clearance de creatinina $92,2 \mathrm{ml} / \mathrm{min} / 1,73 \mathrm{~m}^{2}$. Durante los primeros 5 años del TxR sufrió una fibrilación auricular paroxística (que cedió con amiodarona, la que se prescribió a permanencia), episodios de rinitis alérgica, asma bronquial, reflujo gastroesofágico y lumbago. Al quinto año del TxR la creatinina fue $1,28 \mathrm{mg} / \mathrm{dl}$ y no había proteinuria. Entre el $5^{\circ}$ y $10^{\circ}$ año del TxR la función del injerto se mantuvo estable, aparecieron verrugas vulgares y condilomas acuminados genitales, sufrió una fractura por aplastamiento de un cuerpo vertebral ante un traumatismo menor (osteoporosis), episodios de obstrucción bronquial en relación a infecciones respiratorias altas, hiperplasia de glándulas sebáceas faciales y furunculosis. Al décimo año la creatinina era 1,4 $\mathrm{mg} / \mathrm{dl}$, clearance de creatinina $65 \mathrm{ml} / \mathrm{min} / 1,73 \mathrm{~m}^{2}$, proteinuria $300 \mathrm{mg} / 24 \mathrm{~h}$.

Entre el $10^{\circ}$ y $15^{\circ}$ año del TxR no hubo cambios en la función del injerto, presentó varios episodios asmáticos, se operó de una hernia umbilical y de una colelitiasis sintomática y se diagnosticó una enfermedad ateromatosa difusa de arteria ilíaca izquierda y carotídea, efectuándose un bypass femoro-femoral. La creatinina al año $15^{\circ}$ del TxR fue $1,2 \mathrm{mg} / \mathrm{dl}$, el clearance de creatinina $66,8 \mathrm{ml} /$ $\mathrm{min} / 1,73 \mathrm{~m}^{2}$ y la proteinuria $130 \mathrm{mg} / 24 \mathrm{~h}$. Un año después se diagnosticó una estenosis aórtica calcificada severa con una gradiente sistólica de $68 \mathrm{mmHg}$, con indicación quirúrgica. El paciente decidió operarse sólo en caso de existir riesgo vital. En los 3 años siguientes la función renal se mantuvo estable y la estenosis aórtica no progresó en evaluaciones ecocardiográficas periódicas. También presentó una neuralgia del trigémino autolimitada y sufrió crisis asmáticas de corta evolución en que las radiografías de tórax no mostraron alteraciones pulmonares.

A los 19 años del TxR, una radiografía de tórax tomada con motivo de una evaluación cardiológica mostró infiltrados intersticiales que motivaron un scanner de tórax. Este mostró calcificaciones difusas en ambos pulmones (Figuras 1 y 2) que clínicamente se asociaban a tos seca ocasional y escasa expectoración mucosa matinal (el paciente fumó hasta que ingresó a HD en que suspendió el hábito, 12 paquetes año). Como el paciente no había sufrido de patologías pulmonares que pudieran ocasionar calcificaciones distróficas se interpretó que estas correspondían a CPM. Se solicitó un estudio de función pulmonar y determinaciones

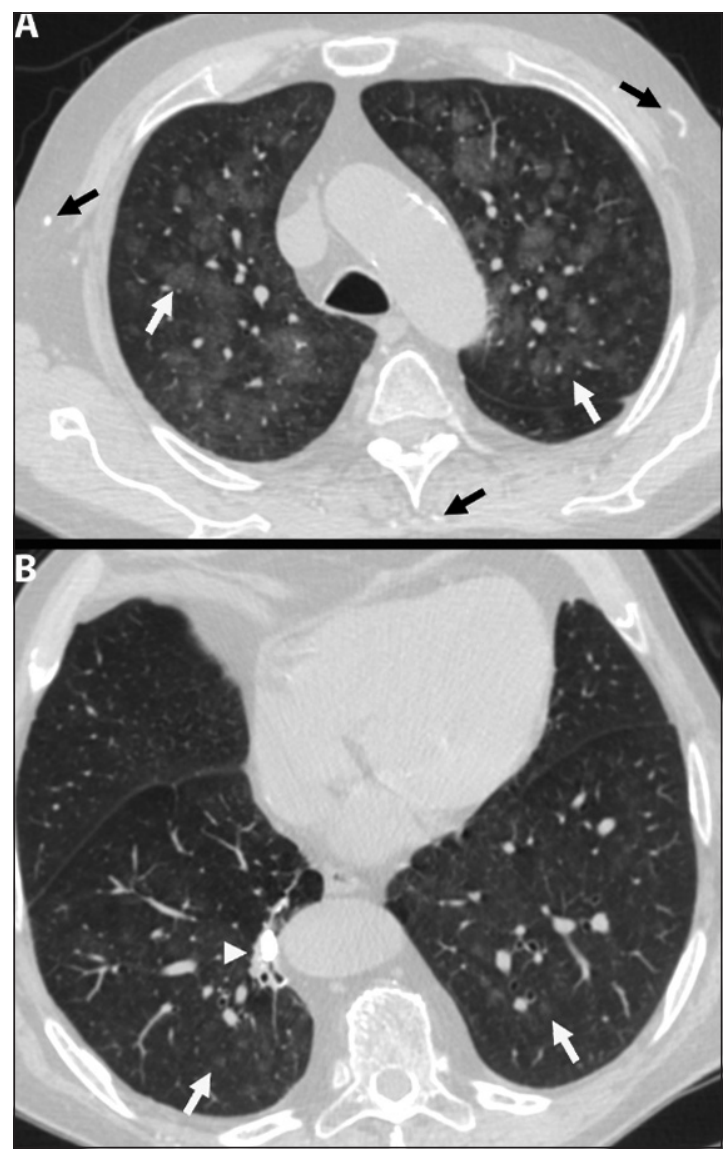

Figura 1. A y B. TC de tórax sin contraste EV. Ventana de parénquima pulmonar. Múltiples nódulos centrolobulillares densos, mal definidos, con tendencia a la confluencia (flechas blancas), en ambos pulmones, más evidentes en ambos lóbulos superiores. Observe la presencia de múltiples calcificaciones parietales vasculares (flechas negras). Presencia de atelectasia de aspecto fibrocicatricial, y granuloma calficado, en segmento basal posterior del lóbulo pulmonar inferior derecho, de aspecto secuelar (cabeza de flecha). 


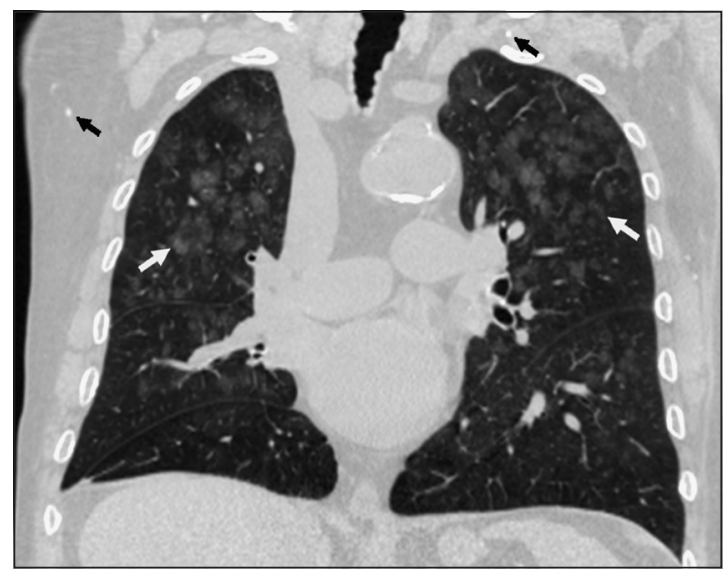

Figura 2. Reconstrucción coronal TC de tórax. Ventana de parénquima pulmonar. Es más evidente el mayor compromiso de ambos lóbulos superiores por estos nódulos centrolobulillares densos confluentes (flechas blancas). Calcificaciones vasculares parietales (flechas negras).

bioquímicas relacionadas al metabolismo del calcio (de las que no se disponían previamente). Sin embargo, el paciente falleció súbitamente de un infarto agudo al miocardio en un servicio de urgencia, semanas después del diagnóstico de CPM y no alcanzó a efectuarlas. Hasta su último control médico, utilizaba prednisona, ciclosporina, ketoconazol, diltiazem, amiodarona, aspirina y losartán. La última creatininemia fue $1,4 \mathrm{mg} / \mathrm{dl}$, la concentración de bicarbonato plasmático 23,2 $\mathrm{mEq} / \mathrm{L}$ y la proteinuria $255 \mathrm{mg} / 24 \mathrm{~h}$.

\section{Discusión}

La CPM es consecuencia del depósito de fosfato de calcio y magnesio en el pulmón ${ }^{16,24}$. La sintomatología respiratoria no se correlaciona con el grado de calcificación macroscópica, como ocurrió con este paciente, que no modificó su tos crónica matinal de ex-fumador y la frecuencia de episodios de crisis asmática. Algunos pacientes con calcificaciones extensas pueden estar asintomáticos y otros con calcificaciones leves o radiografías de tórax normales pueden tener compromiso respiratorio severo ${ }^{26}$.

La causa más común de CPM es la IRC, tanto en etapa pre-dialítica como dialítica ${ }^{3,8,10,15,16,25}$. Algunos pacientes afectados tienen hipercalcemia, hiperfosfemia y una concentración de paratohor- mona elevada con producto calcio/fósforo $>70$ en forma persistente $\mathrm{e}^{7,9,15-17,21,24,27}$, sin embargo, otros no presentan estos trastornos ${ }^{9,11,12,24}$. Aparentemente no existe una correlación entre la duración de la terapia dialítica y la CPM, aunque esto ha sido controvertido ${ }^{12,28}$.

También se ha observado CPM en pacientes trasplantados renales con una mala función del injerto ${ }^{4,22,25}$. Ha sido excepcional que se haya descrito en trasplantados con buena función renal ${ }^{24,29} \mathrm{como}$ lo fue en este paciente operado 19 años antes o en el postoperatorio inmediato de un $\mathrm{TxR}^{14}$.

La radiografía de tórax $(\mathrm{RxTx})$ en los pacientes con CPM habitualmente es normal o muestra infiltrados pulmonares o nódulos que se confunden erróneamente con edema pulmonar, neumonías o embolias pulmonares ${ }^{7,8,10,12,17,21,22,24,25}$. Las calcificaciones usualmente no son visibles ${ }^{19}$ como lo fue en este paciente. Habitualmente los infiltrados predominan en las zonas altas y medias del pulmón $^{9,16,19,24,27}$.

Los exámenes más sensibles para detectar CPM son la tomografía computada de alta resolución (TCAR) y el cintigrama óseo con hidroximetilen-difosfonato marcado con tecnecio99m (Tc99m-MDP) ${ }^{7}$. La TCAR habitualmente muestra opacidades nodulares centrolobulares de 3-10 mm de diámetro en forma difusa, situadas hasta $1 \mathrm{~cm}$ del margen pleural con indemnidad de las regiones costofrénicas. Las calcificaciones pueden ser puntiformes o en anillo envolviendo completamente al nódulo $3,8,11,16,19$. También pueden observarse aéreas de vidrio esmerilado o consolidación pulmonar con distribución lobar ${ }^{3,9,16,17,19}$. Frecuentemente la CPM se asocia a calcificaciones de la aorta y sus ramas, arterias pulmonares segmentarias, calcificaciones en forma de un delgado anillo en las paredes de la tráquea y bronquios y de vasos de la pared torácica, como en este paciente $e^{3,9,19,21 \text {, }}$ ${ }^{25}$ (Figura 1). La RxTx digital de energía dual es más sensible que la RxTx corriente para detectar las calcificaciones, pero su uso aún no está muy difundido $^{28}$. La cintigrafía ósea con Tc99m-MDP es un método muy sensible para detectar calcificaciones pulmonares. En un estudio efectuado en sujetos en HD con RxTx normal, 61\% de ellos mostró calcificación pulmonar estimada por el aumento en la captación del radiofármaco ${ }^{10,24}$. La resonancia magnética es un examen de menor utilidad para la detección de las calcificaciones que los previamente $\operatorname{citados}^{30}$. 
La broncoscopia puede mostrar formaciones lineales blanquecinas de consistencia dura, paralelas entre si y alteraciones nodulares, cuya biopsia muestra que son calcificaciones submucosas ${ }^{4,25}$.

La anatomía patológica muestra depósitos de calcio en las membranas basales alveolares, paredes capilares alveolares, paredes bronquiales y arteriolas pulmonares, sin inflamación pulmonar ${ }^{5}$.

Los tests de función pulmonar, aun en sujetos asintomáticos, muestran reducción de la capacidad pulmonar total y VEF1, disminución de la capacidad de difusión de $\mathrm{CO}$ y reducción de la saturación de oxígeno ${ }^{17,22,24-26}$. En este paciente este estudio no pudo efectuarse por su muerte intempestiva.

El mecanismo por el cual se depositan sales de calcio a nivel pulmonar no está bien aclarado. Se ha postulado que ello es favorecido por el ambiente alcalino de las áreas altas del pulmón, al tener un cociente ventilación/perfusión elevado, que reduciría la solubilidad del calcio y fósforo circulante promoviendo la calcificación ${ }^{5,14}$. También se ha pensado que la acidosis metabólica contribuye a la salida de calcio desde los huesos y que la alcalinidad del período post-diálisis, que aumenta la actividad de las fosfatasas alcalinas favoreciendo la liberación de fosfatos, favorecería el depósito de esos iones en los tejidos blandos ${ }^{31}$. La vitamina D utilizada frecuentemente aumenta la absorción intestinal de calcio y fósforo y las sales de calcio utilizadas como quelantes también facilitarían a la calcificación metastásica y vascular ${ }^{7,32}$. También es posible que el contenido elevado de calcio en las soluciones de HD favorezca la calcificación tisu$\operatorname{lar}^{18}$. En este paciente, con una buena función del injerto renal, no es claro el mecanismo por el cual desarrolló CPM. No es descartable que haya sufrido un hiperparatiroidismo pero ello no alcanzó a estudiarse dado su muerte súbita. La amiodarona, droga que utilizó durante años para la prevención de la recurrencia de una fibrilación auricular, no fue la responsable del daño pulmonar que presentó este paciente, ya que característicamente es del tipo fibrótico y no calcificante.

El tratamiento de las CPM no está establecido. Se han utilizado en su manejo la normalización de las concentraciones sanguíneas de calcio y fósforo, vitamina $\mathrm{D}$, aumento de la dosis de diálisis, reducción de la concentración de calcio en el líquido de dializado, quelantes de fosfatos libres de calcio, cinacalcet, bifosfonatos, paratiroidectomía y el trasplante renal ${ }^{3,5,12,16,22,27,32}$.
Comunicamos este caso por lo poco conocido que es en nuestro medio esta complicación en enfermos con insuficiencia renal crónica y trasplantados renales. Sugerimos que en estos pacientes, frente a la aparición de síntomas respiratorios con una RxTx normal se efectúe un scanner de tórax de alta resolución o una $\mathrm{RxTx}$ digital, ya que sólo estos exámenes radiológicos revelarán la existencia de CPM. Si bien no existe un tratamiento demostrado para esta alteración, la adopción de medidas que intervengan en el metabolismo calcio-fósforo podría ser de utilidad.

\section{Referencias}

1. Bendayan D, Barziv Y, Kramer MR. Pulmonary calcifications: a review. Respir Med 2000; 94: 190-3.

2. Quinibi WY, Nolan CA, Ayus JC. Cardiovascular calcification in patients with end-stage renal disease: a century-old phenomenon. Kidney Int Suppl 2002; 82: s73-80.

3. Lingam RK, The J, Sharma A, Friedman E. Metastatic pulmonary calcification in renal failure: a new HRCT pattern. Br J Radiol 2002; 75: 74-7.

4. Castillo MC, Gimeno MJ, Carro B, Benito JL, Freile E, Sainz JM. Calcinosis pulmonar difusa en paciente con insuficiencia renal. Arch Bronconeumol 2005; 41: 587-9.

5. Chan ED, Morales DV, Welsh CH, Mcdermott MT, Schwarz MI. Calcium deposition with or without bone formation in the lung. Am J Respir Crit Care Med 2002; 165: 1654-69.

6. Rastogi S, Boyers M, Eltorky M, Taneja S. Metastatic pulmonary calcification in a patient with end-stage renal disease on hemodialysis. A common complication but a rare clinical diagnosis. ADV Stud Med 2006; 6: 82-5.

7. Eggert $\mathrm{CH}$, Albright RC. Metastatic pulmonary calcification in a dialysis patient: case report and a review. Hemodial Int 2006; Suppl 2: S51-5.

8. Yatera K, Kawanami T, Ishimoto H, Mukae H. Progressive metastatic pulmonary calcification after successful renal transplantation. Eur Respir Rev 2013; 22: 98-9.

9. Alkan O, Tokmak N, Demir S, Yildirim T. Metastatic pulmonary calcification in a patient with chronic renal failure. J Radiol Case Rep 2009; 3: 14-7.

10. Rosenthal DI, Chandler HL, Azizi F, Schneider PB. Uptake of bone imaging agents by diffuse pulmonary metastatic calcification. AJR Am J Roentgenol 1977; 129: 871-4.

11. Madhusudhan KS, Shad PS, Sharma S, Goel A, Mahajan $\mathrm{H}$. Metastatic pulmonary calcification in chronic renal 
failure. Int Urol Nephrol 2012; 44: 1285-7.

12. Rajkovača Z, Kovačević P, Jakovljević B, Erić Z. Detection of pulmonary calcification in haemodialised patients by whole-body scintigraphy and the impact of the calcification to parameters of spirometry. Bosn J Basic Med Sci 2010; 10: 303-6.

13. Conger JD, Hammond WS, Alfrey AC, Contiguglia SR, Stanford RE, Huffer WE. Pulmonary calcification in chronic dialysis patients. Clinical and pathologic studies. Ann Intern Med 1975; 83: 330-6.

14. Justrabo E, Genin R, Rifle G. Pulmonary metastatic calcification with respiratory insufficiency in patients on maintenence haemodialysis. Thorax 1979; 34: 384-8.

15. Bernard B, McFarlane P, Moid F, Colak E, Perl J. Pulmonary vascular calcification in a nocturnal hemodialysis patient. Clin Nephrol 2012; 77: 231-6.

16. Hochhegger B, Marchiori E, Souza AS, Souza LS, Palermo L. MRI and CT findings of metastatic pulmonary calcification. Br J Radiol 2012; 85: e69-72.

17. Thurley PD, Duerden R, Roe S, Pointon K. Case report: rapidly progressive metastatic pulmonary calcification: evolution of changes on CT. Br J Radiol 2009; 82: e1559.

18. Li YL, Tian YC, Chen YC, Huang SF, Huang CC, Fang JT, et al. Fulminant pulmonary calciphylaxis and metastatic calcification causing acute respiratory failure in a uremic patient. Am J Kidney Dis 2006; 47: e47-53.

19. Marchiori E, Souza AS Jr, Franquet T, Muller NL. Diffuse high attenuation pulmonary abnormalities: a patternoriented diagnostic approach on high resolution CT. AJR Am J Roentegenol 2005; 184: 273-82.

20. Izadyar M, Mahjoub F, Ardakani SN, Ahmadi J. Pulmonary metastatic calcification in a leukemic patient: a case report. J Pediatr Hematol Oncol 2010; 32: e108-10.

21. Hartman TE, Müller NL, Primack SL, Johkoh T, Takeuchi N, Ikezoe J, et al. Metastatic pulmonary calcification in patients with hypercalcemia: findings on chest radiographs and CT scans. AJR 1994; 162: 799-802.

22. Timmins S, Hibbert M. Metastatic pulmonary calcification. N Engl J Med 2010; 363: 2547.

23. Fairshter RD, Vaziri S, Farooqui S, Martin D. Pulmonary pathology in renal transplant recipients. Int J Artif Organs 1984; 7: 327-30.

24. Murris-Espin M, Lacassagne L, Didier A, Voight JJ, Cisterne JM, Giron J, et al. Metastatic pulmonary calcification after renal transplantation. Eur Respir J 1997; 10: 1925-7.

25. Yasuo M, Tanabe T, Komatsu Y, Tsushima K, Kubo K, Takahashi K, et al. Progressive pulmonary calcification after successful renal transplantation. Inter Med 2008; 47: 161-4.

26. Brodeur FJ Jr, Kazerooni EA. Metastatic pulmonary calcification mimicking air-space disease. Technetium99m-MDP spect imaging. Chest 1994; 106: 620-2.

27. Libório AB, Rodrigues L, Freire de Almeida H, Dahler EF. Metastatic pulmonary calcification. NDT Plus 2011; 4: 359-60.

28. Sanders C, Franck MS, Rostand SG, et al. Metastatic calcification of the heart and lungs in end-stage renal disease. Am J Roentgenol 1987; 149: 881-7.

29. Breitz HB, Sirotta PS, Nelp WB, Ott S, Figley MM. Progressive pulmonary calcification complicating successful renal transplantation. Am Rev Respir Dis 1987; 136: 1480-2.

30. Taguhi Y, Fuyund G, Shiova S. MR appearance of pulmonary metastatic calcification. J Computed Assist Tomogr 1996; 41: 38-41.

31. Neff M, Yalcin S, Gupta S, Berger H. Extensive metastatic calcification of the lung in an azotemic patient. Am J Med 1974; 56: 103-9.

32. Yigla M, Keidar Z, Safadi I, Tov N, Reisner SA, Nakhoul F. Pulmonary calcification in hemodialisis patients: correlation with pulmonary artery values. Kidney Int 2004; 66: 806-10. 